\title{
DEFORMATION OF SPHERICAL CAVITIES AND INCLUSIONS IN FLUID-INFILTRATED ELASTIC MATERIALS
}

\author{
J. R. RiCE†, J. W. RUDNICKI‡ and D. A. Simons†
}

\begin{abstract}
(Receired 27 June 1977: in recised form 25 October 1977: receired for publication 16 Nocember 1977)
Abstract-The problem of a spherical cavity which is embedded in a linear, fluid-infiltrated, elastic porous medium and which is subjected to the sudden quasi-static application of a stress at the cavity boundary is solved. It is demonstrated that the deformation of the cavity is homogeneous regardless of the boundary condition imposed on the pore fluid at the cavity wall. For the case in which the pore pressure vanishes at the cavity wall, the time dependence of the cavity strain is evaluated explicitly and is shown to vary hetween the limits of the ordinary linear elastic response based on the short-time (undrained) and on the long-time (drained) properties of the fluid-saturated solid. The results are then used to obtain a relation between the uniform stress or strain applied at infinity and the stress and strain in a highly permeable. possibly non-linear spherical inclusion. The application of this relationship to a study of earthquake premonitory processes based on the deformation of a rock mass with a spherical weakened zone is outlined. It is argued that the fluid coupling effects serve to stabilize the weakened rock against rapid fracture, and give rise instead to a precursory period of accelerating but initially quasi-static straining which ultimately leads to dynamic instability.
\end{abstract}

\section{INTRODUCTION}

Fluid-infiltration of an otherwise elastic porous solid introduces a time dependence into the response to applied loads. For deformation which is much slower than the characteristic time for the diffusion of pore fluid, the local pore fluid pressure in each material element remains constant and the response is said to be drained. Conversely, when load alterations are rapid by comparison to the diffusion time, the local fluid mass content in each material element remains constant, and the response is undrained and elastically stiffer than the drained response. This time dependence has been proposed as a possible factor in accounting for several features of earth-faulting processes: migration of aftershocks [1,2], stabilization of incipient faulting [3], fault creep [4], and premonitory events for earthquakes [5-7]. It is also relevant to a wide range of geotechnical problems including hydraulic fracture [e.g. 3, 8] and soil consolidation $[9,10]$.

An exceptional instance in which the response is time-independent even in the presence of fluid-infiltration is the quasi-static shear of a homogeneous body. If, however, the body contains a cavity or other inhomogeneity, the response is time-dependent. In this paper, we will examine this feature in detail by deriving the solution for the time-dependent strain of a spherical cavity in a fuid-infiltrated elastic porous solid subjected to a suddenly applied shear stress at the cavity boundary. In the course of obtaining the full solution, we will demonstrate that the cavity deforms homogeneously, a result which is analogous to that of Eshelby [11] for the ellipsoidal inclusion embedded in an elastic matrix. The particular relevance of this solution is that it enables us to write an expression which, again analogously with the results of Eshelby [11], relates in a simple way the stress and strain in a spherical inhomogeneity to the applied far-field stress and strain.

By using the results of Eshelby, Rudnicki[12,13] has investigated models for the inception of earth faulting in which the inhomogeneity is considered to be a zone of material weakened by fissuring and past faulting. The present results make it possible to include in these considerations the time-dependent response of the elastic material surrounding the weakened zone. Our analysis suggests that this time-dependent response may be an important factor in leading to an earthquake precursory period of accelerating but initially stable and quasi-static straining prior to instability.

We begin by reviewing the governing constitutive relations and field equations. Then, after deducing the form of the solution from considerations of symmetry and linearity, we establish directly its spatial dependence. Although the solution for the full time dependence involves

tDivision of Engineering, Brown University, Providence, RI 02912, U.S.A.

$\$$ Seismological Laboratory, California Institute of Technology, Pasadend, CA 91125. U.S.A. 
much numerical computation, the time dependence of the strain of the cavity boundary, which is the feature of the solution of greatest interest for applications, will be evaluated explicitly.

\section{GOVERNING EQUATIONS}

The constitutive relations for a linear fluid-infiltrated solid were established by Biot[10]. In order to effect a formulation in terms of easily interpretable parameters, Rice and Cleary [3] exploited the observation that the response of the fluid-saturated solid has the form of the usual linear elastic response in the limits of drained and undrained behavior. For an isotropic linear elastic material the expression for the stress $\sigma_{i j}$ depends on the displacement gradient $u_{i, j} \equiv$ $\left.\partial u_{i} / \partial x_{j}\right)$ and the alteration of pore fluid pressure $p$ as follows:

$$
\sigma_{i j}=\mu\left(u_{i, j}+u_{j, i}\right)+\lambda u_{k, k} \delta_{i j}-\zeta p \delta_{i j}
$$

where $\lambda$ and $\mu$ are the Lame moduli appropriate for drained deformation and $\delta_{i j}$ is the Kronecher delta; $\zeta=1-K / K_{s}^{\prime}$, where $K(=\lambda+2 \mu / 3)$ is the drained bulk modulus and $K_{s}^{\prime}$ may, in certain circumstances, be identified with the bulk modulus of solid constituents, but, more generally, must be regarded as an empirical constant [3]. A second constitutive relations is needed for the alteration of fluid mass (per unit volume) $m$ from its reference value $m_{0}$, and this may be put in the form

$$
m-m_{0}=\zeta \rho_{0}\left[u_{k, k}+\zeta p /\left(\lambda_{u}-\lambda\right)\right]
$$

where $\rho_{0}$ is the reference value of the density of the homogeneous pore fluid and $\lambda_{u}$ is the value of the Lamé constant for undrained deformation. The latter satisfies $\infty>\lambda_{u}>\lambda$, where the upper limit is attained for separately incompressible constituents. For undrained deformation $m=m_{0}$ and inserting

$$
\zeta p=-\left(\lambda_{u}-\lambda\right) u_{k, k}
$$

into (1) verifies that $\lambda_{u}$ is the appropriate Lamé modulus in that case. As suggested in the Introduction, shear of a homogeneous linear isotropic body induces no pore pressure; hence, the shear modulus $\mu$ has the same value for drained and undrained deformation.

In order to complete the formulation of governing equations, we require that the usual equation of stress equilibrium in the absence of body forces be satisfied, i.e.

$$
\sigma_{i j, j}-0
$$

and further, that mass conservation be satisfied for the diffusing pore fluid. As shown in [3]. when the diffusion process obeys Darcy's relation, and when the equilibrium equations and constitutive relations like $(1,2)$ are used to simplify the result, mass conservation implies that $m$ must satisfy the diffusion equation

$$
\nabla^{2} m=\frac{1}{c} \frac{\partial m}{\partial t}
$$

where $c$ is the diffusivity and $\nabla^{2}(\ldots) \equiv \partial^{2}(\ldots) / \partial x_{k} \partial x_{k}$. In this development, Darcy's law

$$
q_{i}=-\rho_{0} \kappa \partial p / \partial x_{i}
$$

relates the mass flow rate in the $x_{i}$ direction, $q_{i}$ (per unit area), to the gradient of pore pressure (in the absence of body force), where $\kappa$ is a permeability which is often given as $\kappa=k / \gamma$ with $k$ in units of area and $\gamma$ the fluid viscosity. The ratio $c / \kappa$ is expressible in terms of elastic moduli in the form

$$
c / \kappa=\zeta^{-2}\left(\lambda_{u}-\lambda\right)\left[(\lambda+2 \mu) /\left(\lambda_{u}+2 \mu\right)\right]
$$


where the term in brackets is the ratio of the moduli governing one dimensional straining under drained and undrained conditions. Other equivalent forms for this expression are available $[3,10]$.

Instead of the fluid mass content $m$ it is more convenient to use

$$
M=\zeta p+\left(\lambda_{u}-\lambda\right) u_{k, k}
$$

which from (2) is proportional to $m-m_{0}$, the alteration of fuid mass content, and thus from (4) satisfies the same diffusion equation, i.e.

$$
\nabla^{2} M=\frac{1}{c} \frac{\partial M}{\partial t}
$$

We note that $M$ has physical units of stress and that in terms of $M$, eqn (1) becomes

$$
\sigma_{i j}=\mu\left(u_{i, j}+u_{j, i}\right)+\lambda_{u} u_{k, k} \delta_{i j}-M \delta_{i j}
$$

Substituting (6) into the equilibrium eqn (3) yields

$$
\left(\lambda_{u}+\mu\right) u_{k, k j}+\mu \nabla^{2} u_{i}-M_{i}=0
$$

which can be recognized as the Navier equations of elasticity with an additional term due to the coupling of the deformation with the pore fluid diffusion.

We wish to solve (5) and (7) subject to a traction derived from a deviatoric stress field $S_{i f}$ $\left(S_{k k}=0\right)$ which is suddenly applied at $t=0$ to the boundary of a spherical cavity of radius $a$. (This problem arises in an obvious way, by superposition, when the actual loading is instead the sudden imposition of a remotely uniform deviatoric stress field $S_{i j}$ on an infinite body containing a spherical cavity. We note that the corresponding problem for purely isotropic remote stress (which is a spherically symmetric problem) has been solved by Rice and Cleary [3]). The alteration of pore fluid pressure is required to vanish at the cavity boundary (although it will be seen that the case of an impermeable boundary is easily treated as well). Thus, the boundary conditions are

$$
\left.\begin{array}{c}
x_{i} \sigma_{i j}=-x_{i} S_{i j} \\
p=0
\end{array}\right\} r=\left(x_{i} x_{i}\right)^{1 / 2}=a, \quad t>0
$$

where the component of the unit normal to the cavity boundary is simply $x_{i} / a$. Because the instantaneous response at the time of load application $(t=0)$ will be undrained, the initial condition is

$$
M\left(x_{i}, t=0\right)=0 .
$$

\section{SOLUTION}

Because of the spherical shape of the cavity and the isotropy of the material, the displacement vector $\mathbf{u}$ can be a function only of the position vector $\mathbf{x}$, the applied stress tensor $\mathbf{S}$, and of scalar quantities such as time $t$ and invariants formed from $\mathbf{x}$ and $\mathbf{S}$, (e.g. $r=(\mathbf{x} \cdot \mathbf{x})^{1 / 2}$. $\mathbf{x} \cdot \mathbf{S} \cdot \mathbf{x}$, etc). Furthermore, because all the equations are linear, the dependence on the applied stress must be linear as well. From these requirements, we could deduce the form of $u$ by using the canonical representation theorems of Wineman and Pipkin [14], but in the present case. it is sufficient simply to observe that the only vector quantities on which $\mathbf{u}$ can depend are $\mathbf{x} \cdot \mathbf{S}$ (with components $x_{k} S_{k i}$ ) and $\mathrm{x}$. Therefore, the displacement must have the form

$$
\mathbf{u}=\mathbf{x} \cdot \mathbf{S} F_{1}(r, t)+\mathbf{x}(\mathbf{x} \cdot \mathbf{S} \cdot \mathbf{x}) F_{2}(r, t)
$$

where the functions $F_{1}, F_{2}$ are to be determined. Note that the quantity $\mathbf{x} \cdot \mathbf{S} \cdot \mathbf{x}$ is a spherical 
harmonic of degree two (i.e. a harmonic function which is homogeneous of degree two in $\mathbf{x}$ ) and the complementary harmonic of negative degree is $r^{-5}(\mathbf{x} \cdot \mathbf{S} \cdot \mathbf{x})$. The latter is employed in the classical elasticity solution for a spherical cavity in an infinite body subjected to uniform shear at infinity [15].

A more convenient representation of $\mathbf{u}$, which is equivalent to (10), is in terms of displacement potentials

$$
\mathbf{u}=\nabla \phi+\mathbf{x} \psi
$$

where $\phi=r^{-5}(\mathbf{x} \cdot \mathbf{S} \cdot \mathbf{x}) F_{3}(r, t)$ and $\psi=r^{-5}(\mathbf{x} \cdot \mathbf{S} \cdot \mathbf{x}) F_{4}(r, t)$ and $(\nabla)_{i} \equiv \partial / \partial x_{i}$. Because the displacement must decay at large distances from the cavity, the $r^{-5}$ has been written explicitly (though at no expense in generality), with the expectation that the solution will involve the same spherical harmonic $r^{-5}(\mathbf{x} \cdot \mathbf{S} \cdot \mathbf{x})$ which appeared in the corresponding problem of classical elasticity. Considerations of symmetry and linearity also indicate that $M$ be written as

$$
M=r^{-5}(\mathbf{x} \cdot \mathbf{S} \cdot \mathbf{x}) g(r, t)
$$

where the function $g(r, t)$ is to be determined.

By using the representations $(11,12)$ we can reduce eqn (7) to two simple equations whose solutions can be obtained by inspection. Substituting (11) into (7) yields

$$
\nabla\left[\left(\lambda_{u}+2 \mu\right) \nabla^{2} \phi+\left(\lambda_{u}+\mu\right)\left(3 \psi+x_{i} \psi_{i}\right)+2 \mu \psi-M\right]+\mu \times \nabla^{2} \psi=0 .
$$

If we form the curl of (13), the gradient expression in the first term vanishes identically and the remainder is

$$
\nabla \times\left(\mathbf{x} \nabla^{2} \psi\right)=-(\mathbf{x} \times \nabla)\left(\nabla^{2} \psi\right)=0
$$

the solution of which is easily shown to satisfy

$$
\nabla^{2} \psi=0
$$

If (14a) is used in (13), integration of the remaining term yields

$$
\left(\lambda_{u}+2 \mu\right) \nabla^{2} \phi+\left(\lambda_{u}+\mu\right)\left(3 \psi+x_{i} \psi_{i}\right)+2 \mu \psi-M=0
$$

where the integration constant may be taken as zero.

The only solution of eqn (14a) which is compatible with the form of $\psi$ following (11) and which decays for large $r$ is

$$
\psi=A r^{5}(\mathbf{x} \cdot \mathbf{S} \cdot \mathbf{x})
$$

where $A$ is at most a function of time.

Substituting (15) and the expression (12) for $M$ into (14b) yields

$$
\left(\lambda_{u}+2 \mu\right) \nabla^{2} \phi=[g(r, t)-2 \mu A] r^{-s}(\mathbf{x} \cdot \mathbf{S} \cdot \mathbf{x})
$$

The solution of (16) is

$$
\phi=B r^{-5}(\mathbf{x} \cdot \mathbf{S} \cdot \mathbf{x})+\left[\frac{\left(\lambda_{u}-\lambda\right)}{(9 \lambda+14 \mu)} f(r, t)+\frac{\mu}{3} A r^{-3}\right] \frac{(\mathbf{x} \cdot \mathbf{S} \cdot \mathbf{x})}{\left(\lambda_{u}+2 \mu\right)} .
$$

The first term is the appropriate solution to the homogeneous equation by the same reasoning as employed in the solution for $\psi$, and $B$ may be a function of time. The first and second terms of the particular solution correspond to the respective terms on the right hand side of eqn (16). The constant multiplying $f(r, t)$ has been chosen for convenience in later calculations and, as 
may be verified by substitution of this expression for $\phi$ into (16), the function $f(r, t)$ is related to $g(r, t)$ of $(12)$ by

$$
f^{\prime \prime}+6 r^{-1} f=r^{-6} \frac{\partial}{\partial r}\left(r^{6} f^{\prime}\right)=\frac{(9 \lambda+14 \mu)}{\left(\lambda_{u}-\lambda\right)} g(r, t) r^{-5}
$$

where $f^{\prime} \equiv \partial f / \partial r$. Integration of this equation yields

$$
f(r, t)=\frac{(9 \lambda+14 \mu)}{\left(\lambda_{u}-\lambda\right)} \int_{\infty}^{t} \rho^{-6} \int_{a}^{\rho} \xi g(\xi, t) \mathrm{d} \xi \mathrm{d} \rho
$$

Although $g(r, t)$ is as yet undetermined. the equation which $g(r, t)$ satisfies is obtained by substituting the expression (12) for $M$ into the diffusion equation (5), and is

$$
\frac{\partial^{2} g}{\partial r^{2}}-\frac{4}{r} \frac{\partial g}{\partial r}=\frac{1}{c} \frac{\partial g}{\partial t}
$$

subject to initial conditions $g(r, t=0)=0, r>a$. We will postpone the solution of (19) and first consider the application of the boundary conditions to eliminate $A$ and $B$.

\section{APPLICATION OF BOUNDARY CONDITIONS AND DEFORMATION OF THE CAVITY SURFACE}

The evaluation of the boundary conditions (8) to determine $A$ and $B$ in $(15,17)$ and the boundary condition on $g(r, t)$ is a long but straightforward algebraic calculation which we will merely outline. By using (11), we can express the boundary conditions (8) in terms of the displacement potentials as follows:

$$
\begin{aligned}
x_{i} \sigma_{i j}= & \mu\left[x_{i} \phi_{. j j}+\frac{1}{2}\left(a^{2} \psi_{. j}+x_{i} x_{i} \psi_{i}\right)\right] \\
& +x_{j}\left(\lambda_{u} \nabla^{2} \phi-M+\mu \psi\right)=-x_{i} S_{i j}, \quad r=a, \\
\zeta p= & M-\left(\lambda_{u}-\lambda\right) \nabla^{2} \phi=0, \quad r=a .
\end{aligned}
$$

The desired conditions are then obtained by substituting (12), (15), and (17) into (20a) and (20b), using (18), and rearranging. We will consider each boundary condition separately.

The traction condition (20a) involves terms multiplying $x_{i}(\mathbf{x} \cdot \mathbf{S} \cdot \mathbf{x})$ and $\mathbf{x} \cdot \mathbf{S}$, and because of the differing orders of $\mathbf{x}$ in these expressions, their coefficients must vanish separately. Thus, from (20a) we obtain two equations which are solved for $A$ and $B$ in terms of $f(a, t)$

$$
\begin{gathered}
A=\frac{15 a^{3}\left(\lambda_{u}+2 \mu\right)}{2 \mu\left(9 \lambda_{u}+14 \mu\right)}[1+b f(a, t)] \\
B=A a^{2} / 5
\end{gathered}
$$

where we have introduced the abbreviation $b=4 \mu\left(\lambda_{u}-\lambda\right) /\left[(9 \lambda+14 \mu)\left(\lambda_{u}+2 \mu\right)\right]$. If these are used in $\phi$ and $\psi$ to compute from (11) the expression for the displacement of the cavity boundary, the result is simply

$$
\mu u_{i}=H(t) S_{i} x_{i}, \quad r=a,
$$

where

$$
H(t)=\frac{3 \lambda_{u}+8 \mu}{9 \lambda_{u}+14 \mu}+f(a, t)\left[\frac{3 \lambda+8 \mu}{9 \lambda+14 \mu}-\frac{3 \lambda_{\mu}+8 \mu}{9 \lambda_{u}+14 \mu}\right]
$$

Equation (22) demonstrates that independently of the boundary condition on the pore fluid. points along the cavity surface displace as if the cavity interior had undergone a homogeneous but time-dependent strain. This is an important feature of the solution, analogous to that 
observed by Eshelby [11] in the case of ordinary elasticity, and we shall exploit it later. We can express $H(t)$ in a more transparent form by recognizing that the expression

$$
\frac{3 \lambda+8 \mu}{9 \lambda+14 \mu}=\frac{4-5 \nu}{7-5 \nu}
$$

(where $\nu$ is Poisson's ratio for drained response), is the factor which appears in the solution of Eshelby for the spherical inclusion. Evidently, $f(a, t)$ increases from zero to unity as the response relaxes from the undrained conditions induced by sudden application of the stress to drained conditions at long times. Correspondingly, $H(t)$ increases from $\left(4-5 v_{u}\right) /\left(7-5 v_{u}\right)$ at $t=0^{+}$to $(4-5 \nu) /(7-5 \nu)$ at $t=\infty$, where $\nu_{u}$ is Poisson's ratio for undrained response and $\nu \leqslant \nu_{u} \leqslant 1 / 2$. For values of Poisson's ratio representative of porous but coherent rocks such as sandstones, say $\nu=0.2$ and $\nu_{u}=0.3, H(t)$ increases by about $10 \%$ from 0.45 to 0.50 . On the other hand, for $\nu=0.2$ and $\nu_{u}=0.4$, which may be more representative of heavily fissured and jointed rock, $H(t)$ increases by $25 \%$, from 0.40 to 0.50 .

In the next section, we will determine the time-dependence of $f(a, t)$ and, thus, the time-dependence of the cavity strain by solving eqn (19) for $g(r, t)$. The condition on $g(a, t)$. corresponding to zero pressure alteration at the cavity boundary, is obtained from (20b) after using the expressions (21) for $A$ and $B$;

$$
g(a, t)=-15 \eta a^{3} \frac{\left(\lambda_{u}-\lambda\right)}{(9 \lambda+14 \mu)}\{1+b f(a, t)\},
$$

where $\eta=\left(\lambda_{u}+2 \mu\right)(9 \lambda+14 \mu) /(\lambda+2 \mu)\left(9 \lambda_{u}+14 \mu\right)$. We note from eqn (18) that $f(a, t)$ is itself defined by a double integral involving the function $g(r, t)$, so the "boundary" condition of eqn (23) is not of a simple kind. It is, nevertheless, typical of those encountered in other coupled problems is porous media (see, e.g. the solutions of Rice and Cleary [3] for radially symmetric problems of cylindrical and spherical cavities).

TIME-DEPENDENCE OF THE CAVITY STRAIN

We wish to solve eqn (19) subject to the boundary condition (23) and the initial condition

$$
g(r, t=0)=0
$$

which follows from (9), the condition that the response is undrained at the instant of load application. For this purpose we introduce the Laplace transform on time

$$
\hat{f}(s)-\mathscr{L}\{f(t)\}=\int_{0}^{\infty} \mathrm{e}^{-s t} f(t) \mathrm{d} t
$$

with inversion by the Bromwich integral[16]

$$
f(t)=\mathscr{L}^{-1}\{\hat{f}(s)\}=\frac{1}{2 \pi i} \int_{C_{B}-i \infty}^{C_{\mathrm{B}}+i \infty} \hat{f}(s) \mathrm{e}^{s t} \mathrm{~d} s
$$

where $i \equiv(-1)^{1 / 2}$ and $C_{B}$ is chosen to be larger than the real part of any singularities in the complex $s$-plane. Applying the Laplace transform to eqn (19) and the boundary condition (23) yields

$$
\begin{gathered}
\left(\frac{\mathrm{d}^{2}}{\mathrm{~d} r^{2}}-\frac{4}{r} \frac{\mathrm{d}}{\mathrm{d} r}-\frac{s}{c}\right) \hat{g}(r ; s)=0, \\
\hat{g}(a ; s)=-15 a^{3} \eta \frac{\left(\lambda_{u}-\lambda\right)}{(9 \lambda+14 \mu)}\left\{s^{-1}+b \hat{f}(a ; s)\right\},
\end{gathered}
$$

where $b$ and $\eta$ have been defined following (21) and (23), respectively. By making the change of 
variables $r=\sqrt{ }(c / s) R$ and writing $\hat{g}(r ; s)=R^{s / 2} G(R ; s)$, we can reduce (24a) to a canonical form of Bessel's equation [17]

$$
G^{\prime \prime}+G^{\prime} R^{-1}-G\left[(5 / 2)^{2} R^{-2}+1\right]=0
$$

where the prime denotes differentiation with respect to $R$. Thus, the solution for $\hat{g}(r ; s)$ which decays for large $r$ is

$$
\hat{g}(r ; s)=C(s) R^{5 / 2} K_{5 / 2}(R)
$$

where $K_{5 / 2}(R)$ is the modified Bessel function of order $5 / 2$ and $C(s)$ is to be determined by the boundary condition. The Bessel functions of half-integer order can be expressed in terms of elementary functions [17] by noting that

$$
K_{1 / 2}(z)=K_{\cdots 1 / 2}(z)=\left(\frac{\pi}{2 z}\right)^{1 / 2} \mathrm{e}^{-z}
$$

and using the recurrence relation

$$
K_{\mu-1}(z)-K_{\mu+1}(z)=-\frac{2 \mu}{z} K_{\mu}(z)
$$

In order to apply the boundary condition $(24 b)$, we express $\hat{f}(a ; s)$ in terms of the solution for $\hat{g}(a ; s)$ by using (18), (26), and the following derivative recurrence relations[17]:

$$
\begin{gathered}
\frac{\mathrm{d}}{\mathrm{d} z}\left[z^{\mu} K_{\mu}(z)\right]=-z^{\mu} K_{\mu-1}(z), \\
\frac{\mathrm{d}}{\mathrm{d} z}\left[z^{-\mu} K_{\mu}(z)\right]=-z^{-\mu} K_{\mu+1}(z) .
\end{gathered}
$$

The result is

$$
\hat{f}(a ; s)=-\frac{C(s)}{5 a^{3}} \frac{(9 \lambda+14 \mu)}{\left(\lambda_{u}-\lambda\right)} q^{3 / 2} K_{3 / 2}(q)
$$

where $q \equiv a(s / c)^{1 / 2}$. Substitution of (27) and (25) into (24b) and the use of (26) yield

$$
C(s)=-15\left(a^{3} / c\right) \eta \frac{\left(\lambda_{u}-\lambda\right)}{(9 \lambda+14 \mu)}\left\{q^{3 / 2} K_{1 / 2}(q)\left[q^{2}+3 \eta(q+1)\right]\right\}^{-1}
$$

The full time dependence of the solution everywhere outside the cavity could be determined by using (28) in (25) and inverting the transform as a function of position. Our main interest, however, is in the time-dependence of the cavity strain, and it is evident from (22) that for this we need only to perform the single inversion for $f(a, t)$. Substitution of $(28)$ into $(27)$ gives

$$
\hat{f}(a, s)=\frac{3 \eta\left(a^{2} / c\right)(1+q)}{q^{2}\left[q^{2}+3 \eta(1+q)\right]}
$$

By factoring the quadratic expression in the denominator, we can rearrange this into terms whose inversions can be found in standard tables of Laplace transform pairs (e.g. [17]). The result is

$$
f(a . t)=1+2[3 \eta(4-3 \eta)]^{-1 / 2}\left[m\left[\beta \exp \left(\beta^{2} \theta\right) \operatorname{erfc}(\beta \vee \theta)\right]\right.
$$


where $\theta=c t / a^{2}, \operatorname{Im}\{\ldots\}$ denotes the imaginary part of $\{\ldots\}, 2 \beta=3 \eta-i[3 \eta(4-3 \eta)]^{1,2}$, and $\operatorname{erf} c(z)$ is the complementary error function

$$
\operatorname{erfc}(z)=1-\operatorname{erf}(z)=\frac{2}{\sqrt{\pi}} \int_{z}^{\infty} \mathrm{e}^{-x^{2}} \mathrm{~d} x
$$

A plot of $f(a, t)$ for two values of $\eta$ is given in Fig. 1. By the expression for $H(t)$ following (22), this same plot represents the time-dependent part of the cavity deformation. (For numerical evaluation, it is more convenient to use the form of $f(a, t)$ which results from direct inversion of the transform by contour integration; this calculation is outlined in the Appendix). If $\lambda=0$ and the constituents are separately incompressible $\left(\lambda_{u}=\infty\right)$, then $\eta=7 / 9$; if $\lambda=\lambda_{k}$, then $\eta=1$. Thus, the cases plotted in Fig. 1 span the practical range of $\eta$ and they indicate that $f(a, t)$ is relatively insensitive to values of the elastic moduli. Fig. 1 also demonstrates that $f(a, t)$ approaches unity, its value for drained response, very rapidly: for $\theta=0.6, f \approx 0.90$.

Asymptotic expressions of (29) for very long and short times can be obtained easily by using the standard expressions [17]:

$$
\operatorname{erfc}(z)=\frac{1}{z \sqrt{ } \pi} \mathrm{e}^{-z^{2}}\left\{1-\frac{1}{2 z^{2}}+\frac{3}{4 z^{4}}-0\left(z^{-6}\right)\right\} \text { as }|z| \rightarrow \infty
$$

and

$$
\operatorname{erfc}(z)=1-\frac{2}{\sqrt{ } \pi} z \mathrm{e}^{-z^{2}}\left\{1+\frac{2}{3} z^{2}+\frac{4}{15} z^{4}+0\left(z^{6}\right)\right\} \text { as }|z| \rightarrow 0
$$

Thus, for short times $\theta \ll 1$,

$$
f(a, t)=(\theta / \pi)^{1 / 2} 6 \eta \mathrm{e}^{-3 \eta(3 \eta / 2-1) \theta}\left\{1-0\left(\theta^{1 / 2}\right)\right\},
$$

and for long times $\theta \gg 1$,

$$
f(a, t)=1-\frac{\theta^{-3 / 2}}{6 \eta \sqrt{ } \pi}-0\left(\theta^{-5 / 2}\right)
$$

In Fig. 2 these expansions are compared with the exact values. Unfortunately, they are good approximations only in a limited range.

Although we have considered the case in which the pore pressure vanishes at the cavity boundary because of its greater potential for applications, we note that the problem in which the cavity wall is impermeable may also be treated. In this case, the boundary condition (20b) is

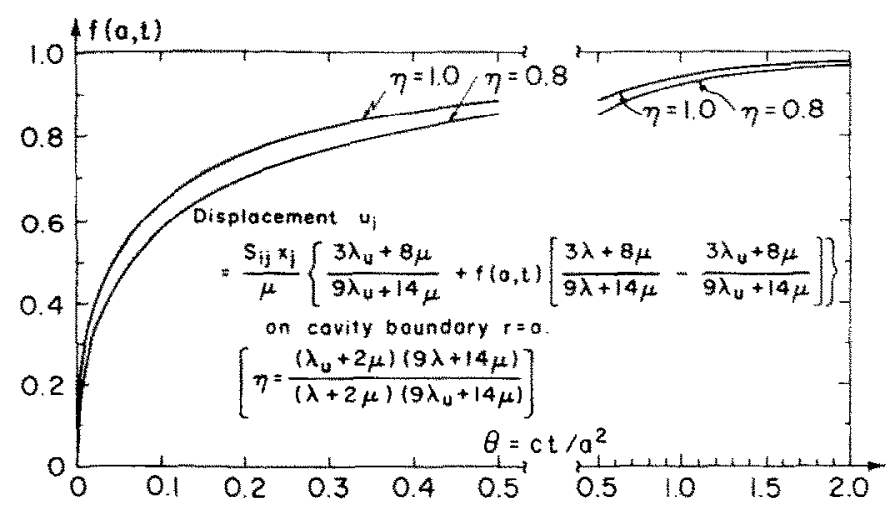

Fig. 1. Time-dependent factor $f(a, t)$ in the expression for displacement of the boundary of a spherical cavity, maintained at zero pore pressure and subjected to suddenly imposed surface tractions derivable from a deviatoric stress tensor $S_{i ;}$. The Lame moduli for drained conditions are $\lambda$ and $\mu$; for undrained conditions they arc $\lambda_{u}$ and $\mu ; c$ is the pore fluid diffusivity. 


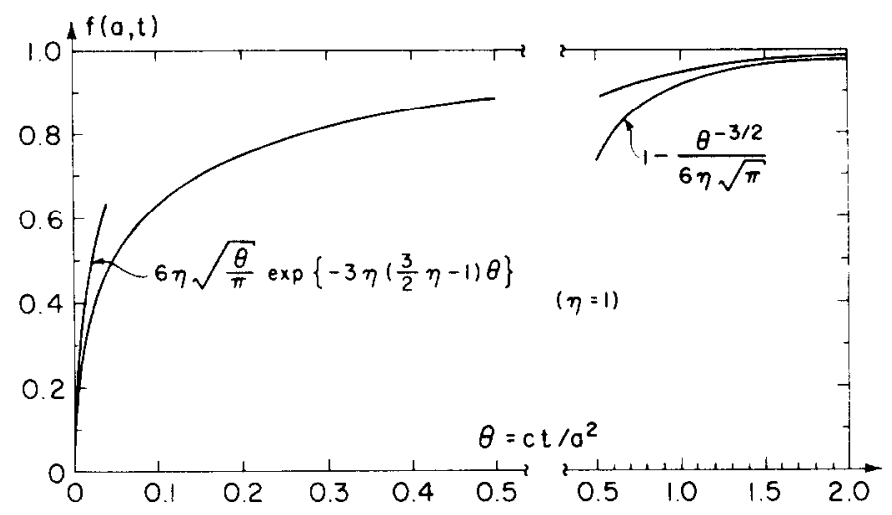

Fig. 2. Comparison of small- and large-time asymptotic expansions with exact calculation (see caption of previous figure).

replaced by the requirement of no fluid mass flow across the cavity boundary,

$$
x_{i} q_{i}=\rho_{0} \kappa x_{i} \frac{\partial p}{\partial x_{i}}=0, \quad r=a,
$$

where we recall that $x_{i}$ is the component of the normal to the cavity boundary. The resulting solution for $\hat{f}(a ; s)$ is

$$
\hat{f}(a ; s)=\frac{9 \eta\left(a^{2} / c\right)(1+q)}{q^{2}\left[q^{3}+4 q^{2}+9 \eta(1+q)\right]} .
$$

While we have not worked out the details of the time-dependence implied by this expression, we note from its limiting values that it too corresponds to a transition from the short-time, undrained to the long-time, drained elastic response of the cavity wall as a function of the time parameter $\theta=c t / a^{2}$.

\section{DEFORMATION OF A SPHERICAL INCLUSION}

We now consider the problem of a possibly nonlinear spherical inclusion which is embedded in a linear, porous, fluid-infiltrated material of the type just considered, and which is deformed by uniform stresses and strains at infinity. Equation (22) and related expressions from [3] will be used to develop a relationship between the stress and strain in the inclusion and the far-field stress and strain. In the next section we will outline the application of this relationship to the study of mechanisms by which pore fluid effects could lend initial stability to earth faulting.

We begin by repeating that eqn (22) demonstrates that the cavity boundary deforms as if the interior had undergone the homogeneous deviatoric strain $\epsilon_{i j}$, where

$$
\mu \epsilon_{i j}=H(t) S_{i j}
$$

Further, if some general time-dependent stress field $S_{i j}(t)$ loads the cavity surface then, by superposition,

$$
\mu \epsilon_{i j}(t)=\int_{-\infty}^{t} H\left(t-t^{\prime}\right) \dot{S}_{i j}\left(t^{\prime}\right) \mathrm{d} t^{\prime}
$$

where the superposed dot denotes a time derivative and $t$ is time.

The corresponding relation for volumetric strain may be taken from the radially symmetric solution of Rice and Cleary[3] for a spherical cavity on the surface of which a total radial compressive stress $\sigma$ is suddenly applied while, simultaneously, a pore pressure $p_{0}$ is applied at the cavity wall. Their results show that for a given total stress $\sigma$ the radial displacement $u$ at 
the cavity wall is independent of $p_{0}$, and has the same form as in the classical elasticity solution. namely

$$
u=3 \sigma a / 4 \mu \text {. }
$$

(This result is not cited explicitly in [3]. It may be proved by evaluating the "hoop strain" u/a, by using eqns $(86,87)$ of $[3]$ for the stresses and pore pressure at the cavity wall, and by using these in the porous medium relation for strain, namely eqn (7) of [3]). Hence the cavity wall deforms as if the interior had undergone a homogeneous volumetric strain $e$ (i.e. one-third of fractional increase in volume), and for a general time varying compressive stress $\sigma(t)$ at the cavity wall we have

$$
\mu e(t)=(3 / 4) \sigma(t)
$$

as the volumetric expression analogous to eqn (30).

Finally, the pressure $p_{0}$ imposed at the wall creates a pore-pressure distribution as given by Rice and Cleary ([3], eqn 86). From that distribution we may calculate by Darcy's law the rate of fluid mass outflow through the cavity wall as

$$
4 \pi a^{2} q(a, t)=-4 \pi a^{2} \rho_{0} \kappa \frac{\partial p}{\partial r}(a, t)=4 \pi \rho_{0} \kappa a\left[1+a\left((\pi c t)^{1 / 2}\right] p_{0}\right.
$$

for $t>0$, where $p_{0}$ is applied at $t=0, q$ is the radial mass flux per unit area, and $k$ is the permeability coefficient introduced earlier. We define $\dot{m}$ by

$$
4 \pi a^{2} q(a, t)+\left(4 \pi a^{3} / 3\right) \dot{m}(t)=0,
$$

so that $\dot{m}$ is the rate, per unit volume, at which the cavity acquires fluid mass. By combining the last two equations we may solve for the function $\dot{m}$ corresponding to the suddenly applied pressure $p_{0}$. Then, by superposition, when an arbitrary pressure history $p(t)$ is applied at the cavity wall the mass accumulation rate per unit volume of cavity is

$$
\dot{m}(t)=-\frac{3 \rho_{0} \kappa}{a^{2}}\left\{p(t)+\int_{-\infty}^{t} \frac{a}{\left[\pi c\left(t-t^{\prime}\right)\right]^{1 / 2}} \dot{p}\left(t^{\prime}\right) \mathrm{d} t^{\prime}\right\}
$$

Equations (30)-(32) have been derived here for a spherical cavity. However, because the cavity wall deforms as if the interior had undergone homogeneous deformation, we may follow the procedure of Eshelby [11] and replace the cavity by an inclusion of any homogeneous material, whether linear or non-linear, with the understanding that the inclusion will deform homogeneously. Now, however, the stipulation that the inclusion be homogeneous also applies to the pore-pressure in the inclusion. Although the pore-pressure cannot, in general, be considered spatially uniform in the inclusion, this will be a good approximation if the inciusion is very much more permeable than the surrounding material. Further, this approximation seems appropriate for our intended applications, in which the inclusion is to represent a zone of heavily fissured, previously faulted material. (Alternatively, although we have no application in mind for it, the case of a non-fluid-infiltrated, impermeable inclusion could also be treated, based on the solution discussed at the end of the previous section).

Suppose then that the material surrounding the inclusion is subjected to the remotely uniform deviatoric stresses $S_{i j}^{\infty}$, mean normal stress $\sigma^{\infty}$, and pore-pressure $p^{\infty}$, whereas the corresponding quantities within the inclusion are $S_{i j}^{\text {ncc }}, \sigma^{\text {inc }}$ and $p^{\text {inc }}$. Let $\epsilon_{i j}^{\infty}$ be the deviatoric strains and $e^{\infty}$ the volumetric strain in the remote surroundings, and let $\epsilon_{i j}^{i n c}$ and $e^{\text {inc }}$ be the corresponding quantities in the inclusion. Then the field in the elastic porous material outside the inclusion can, by superposition, be represented as the sum of the following: (i) a uniform stress/pressure field $S_{\mathrm{ij}}^{\infty}, \sigma^{\infty}, p^{\infty}$ with associated strains $\epsilon_{i j}^{\infty}, e^{\infty}$, and (ii) fields which are identical to those created by loading the wall of a spherical cavity with deviatoric stress $S_{i j}$, compressive 
normal stress $\sigma$, and pore pressure $p$ given by

$$
S_{i j}=S_{i j}^{\infty}-S_{i j}^{\mathrm{inc}}, \quad \sigma=\sigma^{\infty}-\sigma^{\mathrm{inc}}, \quad p=p^{\mathrm{inc}}-p^{\infty},
$$

with associated displacements of the cavity boundary that are consistent with deviatoric strains $\epsilon^{i j}$ and volumetric strain $e$ of the cavity interior, which are

$$
\epsilon_{i j}=\epsilon_{i j}^{\mathrm{inc}}-\epsilon_{i j}^{\infty}, \quad e=e^{\mathrm{inc}}-e^{\infty} .
$$

Of course, the responses of (34) must be related to the loadings of (33) by the expressions (30.31) given earlier. This gives the following two "Eshelby relations" connecting the state within the inclusion to that applied remotely:

$$
\begin{gathered}
\mu\left[\epsilon_{i j}^{\mathrm{inc}}(t)-\epsilon_{i j}^{\infty}(t)\right]=\int_{-\infty}^{t} H\left(t-t^{\prime}\right)\left[\dot{S}_{i j}^{\infty}\left(t^{\prime}\right)-\dot{S}_{i j}^{\mathrm{inc}}\left(t^{\prime}\right)\right] \mathrm{d} t^{\prime} \\
\mu\left[e^{\mathrm{inc}}(t)-e^{\infty}(t)\right]=(3 / 4)\left[\sigma^{\infty}(t)-\sigma^{\mathrm{inc}}(t)\right] .
\end{gathered}
$$

Also, we may now identify $m$ in (32) as the fluid mass content of the inclusion and write

$$
\begin{aligned}
\dot{m}^{\text {inc }}(t)=-\frac{3 \rho_{0} \kappa}{a^{2}} & \left\{\left[p^{\text {inc }}(t)-p^{\infty}(t)\right]\right. \\
& +\int_{-\infty}^{t} \frac{a}{\left.\left[\pi c\left(t-t^{\prime}\right)\right]^{1 / 2}\left[\dot{p}^{\text {inc }}\left(t^{\prime}\right)-\dot{p}^{x}\left(t^{\prime}\right)\right] \mathrm{d} t^{\prime}\right\}}
\end{aligned}
$$

Here we assume tacitly that when $\sigma^{\infty}$ and $p^{\infty}$ vary with time they do so in such a way that $m^{\infty}$ is constant; this is necessary because we have considered the surroundings of the inclusion to be unbounded and to have a remotely uniform state.

Equations (35)-(37) suffice, in principle, as a formulation of the inclusion problem, for if constitutive equations (not necessarily isotropic, linear, or elastic) are given for the inclusion material, relating $S_{i j}^{\text {inc }}, \sigma^{\text {inc }}, p^{\text {inc }}$ to $\epsilon_{i j}^{\text {inc }}, e^{\text {inc }}, m^{\text {inc }}$ then eqns (35)-(37) become a system of integral and albegraic equations enabling the calculation of the time dependent state within the inclusion when the remote stresses $S_{i j}^{\infty}, \sigma^{\infty}, p^{x}$ are specified. (Note that the quantities $\epsilon_{i j}^{x}$ and $e^{x}$ appearing in eqns (35. 36) are related by the usual elastic porous medium stress-strain relations to $S_{i j}^{\infty}, \sigma^{x}, p^{x}$ ).

DISCUSSION: PORE-FLUID EFFECTS IN THE STABILIZATION OF FAULTING

As a focus for discussion of possible pore-fluid effects in the inception of faulting, we adopt a model introduced by Rudnicki[12] and analyzed by him for a solid without fluid effects. In this model the zone which ultimately faults was represented as a weakened inclusion which exhibited, because of previous faulting and fissuring, non-linear inelastic behavior while the surrounding rock, which was stronger, responded in an essentially linear elastic fashion. The weakened zone stress-strain relations were assumed to exhibit peak strengths followed by strain softening and conditions were sought at which the steadily increasing remote shearing stress caused a dynamic "runaway" instability of the inclusion material. Indeed, such strain softening is representative of the failure of brittle rocks (see Jaeger and Cook [19]), although it has been obscured in much of conventional testing by the instability that occurs very near peak strength due to the high elastic compliance of standard testing equipment (see Section 6.13 of [19]).

Consider, for example, the spherical inclusion shown in Fig. 3(a), and suppose that the remote shearing stress $\tau^{\infty}$ induces a pure shear stress $\tau^{\mathrm{inc}}$ in the inclusion. Plots of shear strcss versus engineering shear strain $\gamma$ are shown for the surrounding material (linear, with $\tau^{\infty}=\mu \gamma^{\infty}$ ) and for the inclusion (non-linear) in the figure. We note further that an Eshelby relation 


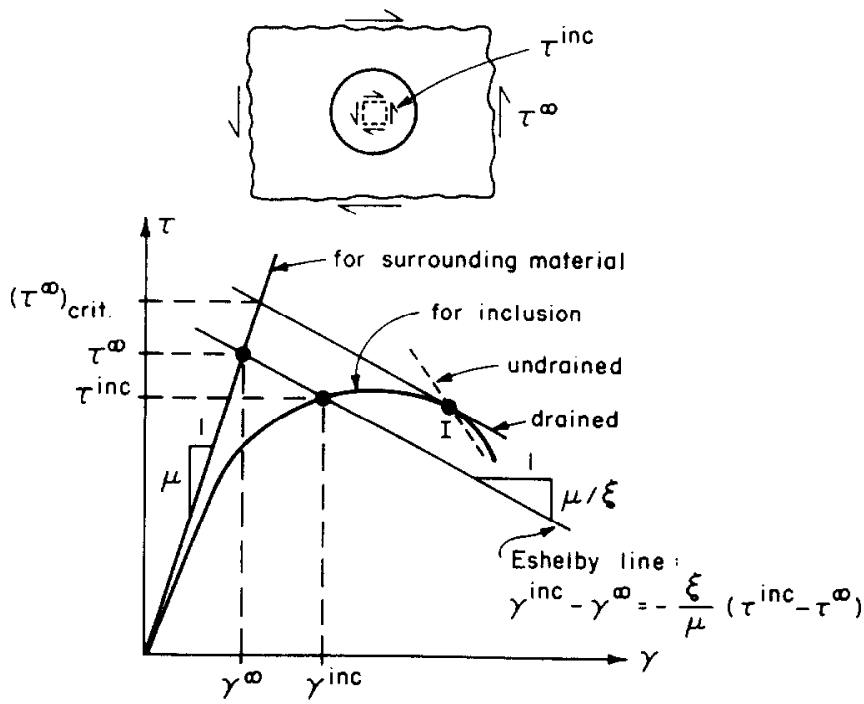

(a)

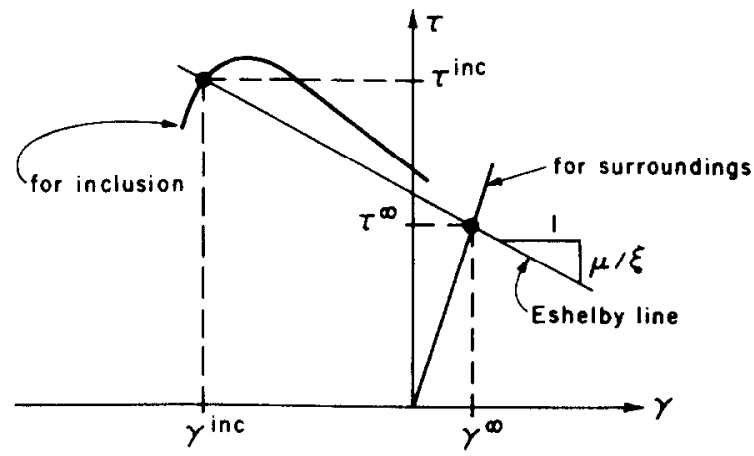

(b)

Fig. 3(a). Shear of a non-linear spherical inclusion (representing a zone weakened by previous faulting) in an unbounded linear material. The greater effective stiffness of the surroundings for rapid, undrained (vs slow, drained) stress alterations means that the system is stabilized against instantaneous failure at point $I$. But the system is "self driving" beyond point I and an initially quasi-static process of strain accumulation, accelerating toward dynamic instability, occurs on time scale controlled by fluid diffusion. (b) "Seismic gap" zone interpretation of inclusion model.

analogous to eqn (35) connects $\tau^{\mathrm{inc}}, \gamma^{\mathrm{inc}}$ to $\tau^{\alpha}, \gamma^{\infty}$, and this has the form

$$
\gamma^{\mathrm{inc}}-\gamma^{\infty}=-(\xi / \mu)\left(\tau^{\mathrm{inc}}-\tau^{\infty}\right)
$$

where it is evident that when fluid effects are negligible and the surroundings respond as if they were drained, we have

$$
\xi=2 H(\infty)=\frac{2(3 \lambda+8 \mu)}{9 \lambda+14 \mu}=\frac{2(4-5 \nu)}{7-5 \nu}
$$

Equation (38) also applies for ellipsoidal inclusions and $\xi$ becomes larger for increasing aspect ratio[11,12]. In fact, a model based on a flattened inclusion may be a much better representation of a natural fault, although the details of time-dependent response can thus far be addressed with some precision only for spherical inclusions.

Specifying $\tau^{\infty}$ and the stress-strain relations for the inclusion and its surroundings enables one to determine the state $\left(\tau^{\text {inc }}, \gamma^{\text {inc }}\right)$ within the inclusion by use of the Eshelby relation of eqn (38) as demonstrated in Fig. 3(a). As $\tau^{\infty}$ is increased the point of contact of the Eshelby line with the inclusion stress-strain curve reaches point $I$ at which the slope is $-\mu / \xi$, and the Eshelby line is tangent to the curve. This is an instability point. Beyond it no quasi-static 
solution exists, and hence point $I$ marks the onset of a dynamic runaway instability. The Eshelby line is, of course, analogous to the unloading characteristic of a testing apparatus; low $\xi$ values correspond to a stiff apparatus, high values to a flexible apparatus.

The failure of laboratory rock systems is often discussed in these terms (e.g. Jaeger and Cook [19]). Stuart[21] represents an existing fault zone by a one-dimensional weakened non-linear strain softening layer situated between two thicker linear elastic layers subjected to shear displacements at their outer boundaries. While not showing a graphical construction in the form of Fig. 3, he arrives at an equivalent characterization of the instability point in terms of the unloading stiffness of the surroundings. Further, as Rice[20] noted, the same representation of instability is valid when the inclusion does not represent a weakened zone, but rather a zone that has lagged the surrounding material in adjustment to tectonic loading so that it presently sustains a higher stress than the remote surroundings. This could, for example, represent a "seismic gap" zone, and the Eshelby line may be used just as before in determining the state within the inclusion (see Fig. 3b). Note that the inclusion stress-strain curve can lie on the negative side of the $\gamma$ axis since the inclusion is misfitting and the zero of $\gamma^{\text {inc corresponds }}$ to the strain into which the inclusion would have to be deformed to just fit into the undistorted surroundings.

The presence of an infiltrating pore fluid modifies the description of failure in Fig. 3 in two ways. The first of these is associated with the time dependent response in the material surrounding the weakened zone and the second with dilatant, pressure-sensitive response of the weakened zone material. For stress alterations which are rapid by comparison to diffusion times, the surroundings respond in an undrained rather than drained fashion. Consequently the response is elastically stiffer as is evident by replacing the parameter $\xi$ in eqn (38), which should now be regarded as relating increments of $\tau$ 's and $\gamma$ 's, by its undrained value which is

$$
\xi_{u}=2 H(0)=\frac{2\left(3 \lambda_{u}+8 \mu\right)}{9 \lambda_{u}+14 \mu}=\frac{2\left(4-5 v_{u}\right)}{7-5 v_{u}}
$$

for a spherical inclusion. This is always lower than the drained value and leads to a steeper "unloading" slope as illustrated by the dashed line through $I$ in Fig. 3(a). (For example, if $\nu=0.2$ and $\nu_{u}=0.4$, we find $\xi=1$ and $\xi_{u}=0.8$ so that there is a $25 \%$ increase in slope). The stiffness ratio $\xi \mid \xi_{u}$ is tabulated for various inclusion shapes by Rice[20] as a function of crack density in the surrounding rock, on the basis of dilute concentration estimates for the effect of microcracks on drained and undrained elastic properties.

Because of this increase in stiffness of the surroundings, for rapid as compared with slow stress alterations, instantaneous failure will not occur at point $I$. The system is instead stabilized transiently by the pore fluid, although it becomes "self-driving" in the sense that, even if $\gamma^{\infty}$ is held constant, $\gamma^{\mathrm{inc}}$ continues to increase on a time scale controlled by fluid diffusion. Ultimate dynamic instability results when (and, indeed, if) $\gamma^{\text {inc }}$ has increased enough for the softening slope to fall to $-\mu / \xi_{u}$. This failure process is goverened by the following non-linear integral equation based on eqn (35):

$$
\gamma^{\text {inc }}(t)-\gamma^{\infty}(t)=\frac{2}{\mu} \int_{-\infty}^{t} H\left(t-t^{\prime}\right)\left[\dot{\tau}^{\infty}\left(t^{\prime}\right)-\tau^{\text {inc }}\left(t^{\prime}\right)\right] \mathrm{d} t^{\prime}
$$

where $\gamma^{\infty}=\tau^{\infty} / \mu$ and where $\tau^{\text {inc }}$ may be expressed as a function of $\gamma^{\text {inc }}$ as, e.g. in the plot of Fig. 3(a). Of course, the same discussion applies for the seismic gap model of Fig. 3(b).

In the context of earth faulting $\tau^{\infty}$ increases very slowly with time through large scale tectonic processes. Thus it is normally to be expected that, except for inclusion states in the near vicinity of point $I$, the response of the system should be very nearly drained. This is so even if pore pressures considerably above hydrostatic should persist over a large region including the inhomogeneity, for these may continuously equilibrate within the region, with the effect only of raising the reference pressure with respect to which the alterations, denoted herein by $p$, take place. Then as point $I$ is passed subsequent deformation of the system is self-driving. The ensuing rupture process is a potential source of detectable earthquake precursors. In particular, our considerations suggest a precursory period, comparable in 
duration to diffusive relaxation times (say, $\approx 0.1 a^{\%} / \mathrm{c}$ from Fig. 1), over which accelerating deformation takes place within the soon to be faulted zone. Because deformation during this period is much more rapid than large scale tectonic processes, it may be accompanied by observable precursory effects, such as tilting and creeping of the ground surface at anomalously high rates, changes in travel times of seismic waves as suggested in "dilatancy-diffusion" models [5-7], and perhaps other effects. Rice [20] has given some approximate extimates of the time scale involved in precursory processes of the kind described, based on a specific $\tau$ vs $\gamma$ relation for the inclusion and on the replacement of the actual $H(t)$ in the above integral equation by that for a standard linear model with relaxation time of $0.1 \mathrm{a}^{2} / \mathrm{c}$. Although the choice of material and geometric parameters is very uncertain, it does seem plausible from the results that precursory periods arising from the mechanism described could have time scales comparable to those of observed precursors (Scholz et al.[6]) to smaller earthquakes.

The second possible mode of pore fluid stabilization of a fault zone is complementary to that just discussed and arises because of the tendency for rock, at least in initially coherent laboratory specimens, to exhibit dilatant deformation as it is sheared. When the rock is fluid-infiltrated and when the time scale of stress alterations does not allow full drainage by diffusion, $p^{\text {inc }}$ is decreased relative to $p^{\infty}$. This serves to increase the Terzaghi effective compressive stresses within the weakened zone, and hence strengthens the zone in its resistance to shear. The phenomenon is referred to as "dilatant hardening." A preliminary analysis has been given by Rudnicki[13] based on expressions such as eqns (36)-(38) implemented with a coupling between deformation-induced pore-pressure alterations and pressure-sensitive dilatant constitutive descriptions in the manner of Rice [18]. Like the first effect discussed, dilatant strengthening is also expected to become important only in the vicinity of point $I$ in Fig. 3, and to have similar effects in stabilizing the rock against instantaneous failure. giving rise to a precursory period of accelerating but initially quasi-static deformation.

Both of these possible stabilizing effects of the pore fluid need to be more fully studied. We have given a basic formulation for doing so here, through the Eshelby relations given as eqns (35)-(37). It is clear, however, that the evolution of the rupture process will depend strongly on features of the inelastic constitutive response of the weakened zone which are themselves not well understood, and that predictions may be very much affected by the shape of such zones, which are likely to be more flattened than spherical.

Acknowledgements - The study was supported by the NSF Geophysics Program and by the USGS Farthquake Hazards Reduction Program. We are grateful to M. P. Cleary for helpful discussions.

\section{REFERENCES}

1. A. Nur and J. R. Booker, Aftershocks caused by fluid flow. Science 175, 885 (1972).

2. J. R. Booker, Time-dependent strain following faulting of a porous medium. J. Geophys. Res. 79. 2037-2044 (1974).

3. J. R. Rice and M. P. Cleary, Some basic stress-diffusion solutions for fluid-saturated elastic porous media with compressible constituents. Rev. Geophys. Space Phys. 14, 227-241 (1976)

4. J. R. Rice and D. A. Simons, The stabilization of spreading shear faults by coupled deformation-diffusion effects in fluid-infiltrated porous materials. J. Geophys. Res, 81, 5322-5334 (1976).

5. A. Nur, Dilatancy, pore fluids, and premonitory variations of $t_{s} / t_{p}$ travel times. Bull. Seismol. Soc. Am. 62, $1217-1222$ (1972).

6. C. H. Scholz L. R. Sykes, and Y. P. Aggarwal, Earthquake prediction: a physical basis. Science 181, $803-810$ (1973).

7. D. L. Anderson and J. H. Whitcomb. Time-dependent seismology. J. Geophys. Res. 80, 718-732 (1975).

8. B. Haimson and C. Fairhurst, Insitu stress determination at great depth by means of hydraulic fracturing. In Rock Mechanics-Theory and Practice. (Edited by W. H. Somerton), 559-584. Society of Mining Engineers, The American Institute of Mining, Metallurgical and Petroleum Engineers, Inc., Salt Lake City, Utah (1959).

9. J. McNamee and R. E. Gibson, Displacement functions and linear transforms applied to diffusion through porous elastic media. Quart. J. Mech. App. Math. 13,99-111 (1960).

10. M. A. Biot, General theory of three dimensional consolidation. J. App. Phys. 12, 155-164 (1941).

11. J. D. Eshelby, Elastic inclusions and inhomogeneities." In Progress in Solid Mechanics (Edited by I. N. Sneddon and R. Hill), Vol. 2, 88-140, North-Holland, Amsterdam (1961).

12. J. W. Rudnicki, The inception of faulting in a rock mass with a weakened zone. J. Geophys. Res. 82, 844-854 (1977).

13. J. W. Rudnicki, Localization of deformation, brittle rock failure, and a model for the inception of earth faulting. Ph.D. Thesis, Brown University, Providence, R.I. (1977).

14. A. S. Wineman and A. C. Pipkin, Material symmetry restrictions on constitutive equations. Arch. Rat. Mech. Anal. 17. 184-214 (1964).

15. A. E. H. Love, A Treatise on the Mathematical Theory of Elasticity. Dover, New York (1944).

16. G. F. Carrier, M. Krook and C. E. Pearson, Functions of a Complex Variable Theory and Technique. McGraw-Hill, New York (1966) 
17. M. Abramowitz and I. A. Stegun (editor)., Handbook of Mathematical Functions, National Bureau of Standards, Applied Mathematics Series 55, Washington (1964).

18. J. R. Rice, On the stability of dilatant hardening for saturated rock masses. J. Geophys. Res. 80, 1531-1536 (1975).

19. J. C. Jaeger and N. G. W. Cook, Fundamentals of Rock Mechanics, 2nd Edn. Chapman \& Hall, New York (1976).

20. J. R. Rice, "Theory of precursory processes in the inception of earthquake rupture," Proc. Sympos. on Physics of Earhquake Sources (at 1977 General Assembly of Int. Assoc. of Seismology and Physics of the Earth's Interior. Durham, England), (Edited by V. Myachkin), German Democratic Republic Academy of Sciences Publication-in press.

21. W. D. Stuart. Diffusionless dilatancy model for earthquake precursors. Geophys. Res. Lett. 1. 261-264 119741.

\section{APPENDIX}

We write $h(t)$ for the function $f(a, t)$ and outline here the direct inversion of

$$
\hat{h}(s)=\frac{3 \eta\left(a^{2} / c\right)(1+q)}{q^{2}\left[q^{2}+3 \eta(1+q)\right]}
$$

where $q=a(s / c)^{1 / 2}$, by the Bromwich integral

$$
h(t)=\frac{1}{2 \pi i} \int_{B r} \hat{h}(s) \mathrm{e}^{s t} \mathrm{~d} s,
$$

where the integration is along $A B$ in Fig. 4. It is convenient to let $z=s a^{2} / c\left(=a^{2}\right)$ and $\theta=c t / a^{2}$ so that

$$
h(\theta)=\frac{3 \eta}{2 \pi i} \int_{B r} \frac{\left(1+z^{1 / 2}\right) \mathrm{e}^{z \theta} \mathrm{d} z}{z\left[z+3 \eta\left(1+z^{1 / 2}\right)\right]}
$$

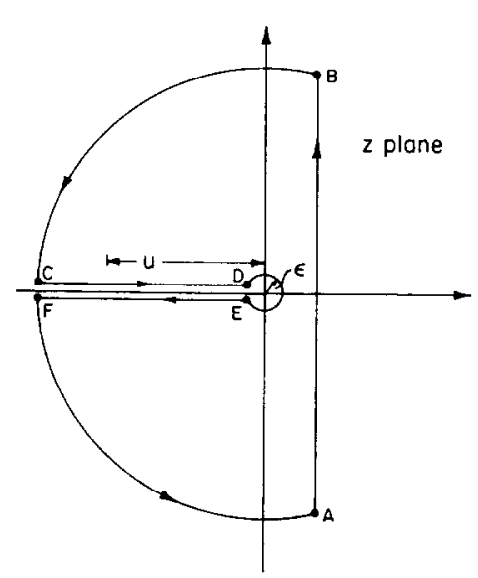

Fig. 4. Contour for inversion of transform.

The branch cut for $z^{1 / 2}$ is taken along the negative real axis. The bracketed term in the denominator of the integrand vanishes when $z^{1 / 2}=-3 \eta / 2 \pm i\left(12 \eta-9 \eta^{2}\right)^{1 / 2} / 2$. but because these roots have negative real parts. the corresponding values of $z$ do not fall in the $z$-plane with the branch cut as just described. Thus the integrand is analytic within the closed contour $A B C D E F A$, and by Cauchy`s integral theorem [16] it may be written

$$
\frac{1}{2 \pi i} \oint\{\ldots\} \mathrm{d} z=h(\theta)+\frac{1}{2 \pi i} \int_{B C D E F A} \hat{h}(z) \mathrm{e}^{z \theta} \mathrm{d} z=0
$$

where $h(\theta)$ is the contribution from $A B$. Setting $:=R e^{i \phi}$ on $B C$ and $A F$ and letting $R \rightarrow x$ demonstrates that there is also no contribution from these segments. If we let $z=\epsilon \mathrm{e}^{i \phi}$ on $D E$ and take the limit $\epsilon \rightarrow 0$. this integral contributes -1 . On $C D$ we set $z=u \mathrm{e}^{i \pi}$ and on $E F, z=u \mathrm{c}^{-i \pi}$ so that the branch line integrals become

$$
\frac{1}{\pi} \int_{0}^{x} \ln \left\{\frac{1+i u^{1 / 2}}{u\left[u-3 \eta\left(1+i u^{1 / 2}\right)\right]}\right\} \mathrm{e}^{i u \theta} \mathrm{d} u
$$

Thus. the formula for $h(\theta)$ becomes

$$
h(\theta)=1-\frac{6 \eta}{\pi} \int_{1)}^{x} \frac{\mathrm{e}^{-X^{2} \theta} X^{2} \mathrm{~d} X}{X^{4}+3 \eta(3 \eta-2) X^{2}+9 \eta^{2}}
$$

where we have set $u=X^{2}$ in the integral. The curves in Figs. 1 and 2 have been calculated by numerical evaluation of this integral. 